\title{
DIFFERENT LINEAR AND NON-LINEAR FORM OF TRAPEZOIDAL NEUTROSOPHIC NUMBERS, DE-NEUTROSOPHICATION TECHNIQUES AND ITS APPLICATION IN TIME-COST OPTIMIZATION TECHNIQUE, SEQUENCING PROBLEM
}

\author{
Avishek Chakraborty ${ }^{1,4}$, Sankar Prasad Mondal $^{2, *}$, Animesh Mahata $^{3}$ and \\ SHARIFUL Alam ${ }^{4}$
}

\begin{abstract}
In this research article, we envisage the neutrosophic number from various distinct rational perspectives \& viewpoints to give it a look of a conundrum. We focused \& analysed various types of linear and non-linear generalized trapezoidal neutrosophic numbers which serves an indispensable role for uncertainty concept related problem. We also introduce the idea of de-neutrosofication for trapezoidal neutrosophic number using an influx of different logical \& innovative methods by which we move with a manifesto to convert a neutrosophic number into a crisp number. Using this concept of de-neutrosophication, we analyze some real-life problem like networking Crash model problem and job-sequencing problem of operation research field when the numbers are in trapezoidal neutrosophic ambience. We also compare our specified result with previously defined score and accuracy function and try to present some interesting and better result without any possible fiasco. This noble thought will help us to solve a plethora of daily life problems in neutrosophic arena.
\end{abstract}

Mathematics Subject Classification. 46N10, 68T37.

Received May 26, 2019. Accepted September 9, 2019.

\section{INTRODUCTION}

The concept of vagueness \& uncertainty in this field was first portrayed by Zadeh in his paper [44]. Moving on with the paper of Zadeh, Atanassov [2] in the year 1986 presents the legerdemain idea of an intuitionistic fuzzy set in the field of Mathematics in which he considers the concept of membership function as well as nonmembership function in case of intuitionistic fuzzy set. With the invention of Liu and Yuan in [25], he ignited the concept of triangular intuionistic fuzzy set, which in reality is the congenial mixture of triangular fuzzy set and intuionistic fuzzy set. Later, Ye [39] introduced the elementary idea of trapezoidal intuitionistic fuzzy set

Keywords. Linear and non-linear Neutrosophic number, de-neutrosophication methods, time-cost optimization technique, sequencing problem.

1 Department of Basic Science, Narula Institute of Technology, Agarpara, 700109 Kolkata, India.

2 Department of Applied Science, Maulana Abul Kalam Azad University of Technology, West Bengal, Haringhata, Nadia, West Bengal, India.

3 Mahadevnagar High School, Mahestala, 700141 Kolkata, West Bengal, India.

4 Department of Mathematics, Indian Institute of Engineering Science and Technology, Shibpur, 711103 Howrah, India.

*Corresponding author: sankar.res07@gmail.com 
where both truth function and falsity function are both trapezoidal number in nature instead of triangular. The uncertainty theory plays an influential role to create some interesting model in various fields of science and technological problem. However, there is an elementary question will arise: how can we inchoate or use the uncertainty assumptions in our mathematical modeling related with daily life? Researchers, everywhere from the universe invented lots of approaches \& methods to define them and they gave their different kind of recommendation to use the uncertainty philosophy. There are different types of suggestions in literature to classify some basic ambiguous or garrulous parameters. It should be esteemed that there is no unique representation of the uncertain parameter. For solving a problem, the decision maker's choice can be variously conferred in different applications. Smarandache in 1995 (published in 1998) [34] manifested the idea of neutrosophic set where there are three different components namely (i) truth, (ii) indeterminacy, (iii) falsehood. All the aspect of neutrosophic set is very much pertinent with our real life system. For example, in a given hypothesis "X party will emerge victorious in this parliamentary election" in order to represent the class of voters who immediately agree with the proposition falling under the idea of truth (truth part) whereas there will be some of them who will deny the proposition, which serves as the falsity part and there are some people with minds facing hesitation which serves the part of indeterminacy. Obviously, neutrosophic concept is a very effective \& an exuberant idea in these cases. Ye [41] introduced trapezoidal neutrosophic set and developed some score and accuracy function in this domain. He also utilizes this trapezoidal neutrosophic concept in multi criteria decision making problem. Recently, Avishek et al. [8] represents a triangular neutrosophic fuzzy set in different types of form and perform de-neutrosophication in case of triangular neutrosophic number.

However, we will give some information about the uncertain parameter, and displayed how it differs and varies from other thoughts of uncertainty using definitions, flowcharts, algorithm and diagrams. In this paper, we suggest the researchers to take the uncertain parameters as trapezoidal neutrosophic parameter. We developed the idea of de-neutrosophication method for trapezoidal neutrosophic case using area removal method and graded mean method which can convert a trapezoidal neutrosophic number into a crisp number. Using that deneutrosophic value we solved two different problem (i) crash model in networking field and (ii) job-sequencing problem, where, representation of the numbers are in trapezoidal neutrosophic type.

\section{Some basic difference between some uncertain parameter}

We consider an Interval number and then we can see:

(i) The data belongs to a particular interval;

(ii) There was no approach of membership function.

We consider a Fuzzy number and then we can see:

(i) The conception of belongingness is arising.

(ii) The need of membership function arises.

We consider an Intuitionistic fuzzy number and then we can see:

(i) The conception of membership function and non-membership function are arises.

(ii) The use of belongingness and non-belongingness of the function is present.

We Consider a Neutrosophic fuzzy number and then we can see:

(i) The idea of truthiness, falsity and indeterminacy of the elements are arises;

(ii) The utility of membership function for truthiness, falsity and indeterminacy will arise.

The idea is given bellow as: 


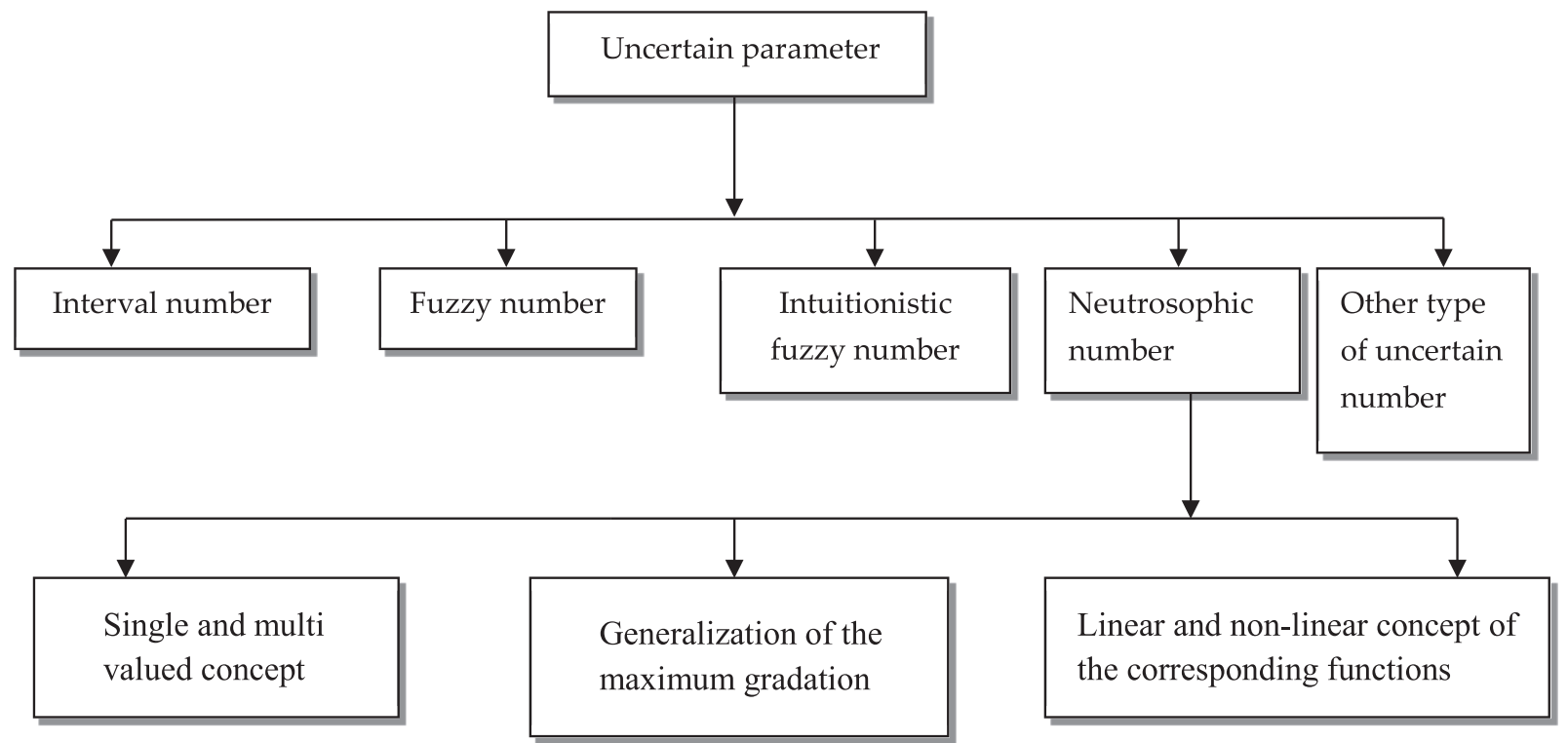

Fuzzy set can't deal properly with a situation where the decision is improper and the decision maker's statement is ambiguous. Generalizations of fuzzy set theory is taken to be one of Intuitionistic fuzzy set theory (IFS). The fuzzy set considers only the degree of belongingness but not the non belongingness. Fuzzy set theory does not incorporate the degree of hesitation (i.e., degree of non-determinacy defined. To handle such facts, Atanassov [2] explored the concept of fuzzy set theory by IFS theory.

For that reason, we need to develop some new ideas for solving the uncertainty occurring problem. The idea of Neutrosophic Sets (NSs) [34] considers the truth membership function, the indeterminacy membership function, and the falsity membership function simultaneously, which are more constructive and as well as applicable arather than the general fuzzy set concept and intuitionistic fuzzy set concept. Neutrosophic number is mainly dealing with uncertain, incomplete and inconsistent information. With the advancement of research day by day we see that Single-Valued Neutrosophic Set concept is introduced and basically it is the extension part of neutrosophic set Wang et al. [36]. Ye [40] introduced the concept of simplified Neutrosophic Sets, and Peng et al. $[28,30]$ developed some ideas on novel operations and aggregation operators. Recently, researchers give their attention on neutrosophic set they developed different kind of extensions of Neutrosophic set, such as Interval Neutrosophic Set [36], Bipolar Neutrosophic Sets [40] and Multi-valued Neutrosophic Sets [28,30].

Decision making theory is very important tool for decision makers. The problem may be comes with either crisp $[3,9,10,43]$ or uncertain data. The uncertainty theory and decision making problem is an important topic now a days. The developments are still going on the field of fuzzy decision-making problem $[7,11,12,18,19,21,37]$, intuitionistic fuzzy decision-making problem $[24,33]$ and in neutrosophic decision making problem $[1,4,15,17,20$, $22,23,26,27,29,31,32,35,38,42,45]$. The decision-making problem with uncertainty has expand its application in operation research [8], graph theory $[5,6]$ etc. Recently, in neutrosophic arena $[13,14,16]$ expanded in its different applicability in scientific application.

The basic idea and new work is done in this article are shown here:

(1) Defining the uncertainty using different type of neutrosophic number.

Here we define the concept of generalized trapezoidal nutrosophic number and nonlinear trapezoidal neutrosophic number in different forms.

(2) Finding the de-neutrosophication value.

Using the concept of (i) Removal area method and (ii) Mean interval method we find the deneutrosophication value. Also we compare the uncertainties between triangular and trapezoidal neutrosophic number. 
(3) Application in different fields.

We apply the concept in time cost uncertain optimization problem and uncertain sequencing problem.

\section{Neutrosophic Number}

Definition 2.1 (Neutrosophic Set [34]). A set $\widetilde{n S}$ in the universal discourse $X$, symbolically denoted by $x$, it is called a neutrosophic set if $\widetilde{n S}=\left\{x ;\left[\tau_{\widetilde{n S}}(x), \iota_{\widetilde{n S}}(x), \varepsilon_{\widetilde{n S}}(x)\right] \vdots x \in X\right\}$, where $\tau_{\widetilde{n S}}(x): X \rightarrow[0,1]$ is said to be the truth membership function, which represents the degree of confidence, $\iota \widetilde{n S}(x): X \rightarrow[0,1]$ is said to be the indeterminacy membership, which represents the degree of uncertainty, and $\varepsilon_{\widetilde{n S}}(x): X \rightarrow[0,1]$ is said to be the falsity membership, which represents the degree of skepticism on the decision taken by the decision maker. $\tau_{\widetilde{n S}}(x), \iota_{\widetilde{n S}}(x) \& \varepsilon_{\widetilde{n S}}(x)$ exhibits the following relation:

$$
-0 \leq \tau_{\widetilde{n S}}(x)+\iota_{\widetilde{n S}}(x)+\varepsilon \widetilde{n S}(x) \leq 3+.
$$

Definition 2.2 (Single-Valued Neutrosophic Set). A Neutrosophic Set $\widetilde{n S}$ in the Definition 2.1 is said to be a Single-Valued Neutrosophic Set $(\widetilde{S n S})$ if $x$ is a single-valued independent variable. $\widetilde{S n S}=$ $\left\{x ;\left[\tau_{\widetilde{S n S}}(x), \iota_{\overparen{S n S}}(x), \varepsilon_{\widetilde{S n S}}(x)\right] \vdots x \in X\right\}$, where $\tau_{\widetilde{S n S}}(x), \iota \widetilde{S n S}(x) \& \varepsilon_{\widetilde{S n S}}(x)$ denoted the concept of accuracy, indeterminacy and falsity memberships function respectively.

If there exist three points $a_{0}, b_{0} \& c_{0}$, for which $\tau_{\widetilde{S n S}}\left(a_{0}\right)=1, \iota \widetilde{\operatorname{SnS}}\left(b_{0}\right)=1 \& \varepsilon_{\widetilde{S n S}}\left(c_{0}\right)=1$, then the $\widetilde{S n S}$ is called neut-normal.

$\widetilde{S n S}$ is called neut-convex, which implies that $\widetilde{S n S}$ is a subset of a real line by satisfying the following conditions:

(i) $\tau_{\widetilde{S n S}}\left\langle\delta a_{1}+(1-\delta) a_{2}\right\rangle \geq \min \left\langle\tau_{\widetilde{S n S}}\left(a_{1}\right), \tau_{\widetilde{S n S}}\left(a_{2}\right)\right\rangle$

(ii) $\iota_{\overparen{S n S} S}\left\langle\delta a_{1}+(1-\delta) a_{2}\right\rangle \leq \max \left\langle\iota_{\overparen{S n S}}\left(a_{1}\right), \iota_{\overparen{S n S}}\left(a_{2}\right)\right\rangle$

(iii) $\varepsilon_{\overparen{S n S}}\left\langle\delta a_{1}+(1-\delta) a_{2}\right\rangle \leq \max \left\langle\varepsilon_{\overparen{S n S}}\left(a_{1}\right), \varepsilon_{\overparen{S n S}}\left(a_{2}\right)\right\rangle$,

where $a_{1} \& a_{2} \in \mathbb{R}$ and $\delta \in[0,1]$.

Definition 2.3 (Single-Valued Trapezoidal Neutrosophic Number [39]). A Single-Valued Neutrosophic Number $(\tilde{z})$ is defined as $\tilde{z}=\left[\left(p^{1}, q^{1}, r^{1}, s^{1}\right) ; \mu\right],\left[\left(p^{2}, r^{2}, r^{2}, s^{2}\right) ; \vartheta\right]$, [( $\left.\left.p^{3}, q^{3}, r^{3}, s^{3}\right) ; \varphi\right]$, where $\mu, \vartheta, \varphi \in[0,1]$. The accuracy membership function $\left(\tau_{\tilde{z}}\right): \mathbb{R} \rightarrow[0, \mu]$, the indeterminacy membership function $\left(\iota_{\tilde{z}}\right): \mathbb{R} \rightarrow[\vartheta, 1]$ and the falsity membership function $\left(\varepsilon_{\tilde{z}}\right): \mathbb{R} \rightarrow[\varphi, 1]$ are given as:

$$
\begin{aligned}
& \tau_{\tilde{z}}(x)=\left\{\begin{array}{ll}
\tau_{\widetilde{z l}}(x) & p^{1} \leq x \leq q^{1} \\
\mu & q^{1} \leq x \leq r^{1} \\
\tau_{\widetilde{z u}}(x) & r^{1} \leq x \leq s^{1} \\
0 & \text { otherwise }
\end{array}, \quad \iota_{\tilde{z}}(x)= \begin{cases}\iota_{\widetilde{z l}}(x) & p^{2} \leq x \leq q^{2} \\
\vartheta & q^{2} \leq x \leq r^{2} \\
\iota_{\widetilde{z u}}(x) & r^{2} \leq x \leq s^{2} \\
1 & \text { otherwise }\end{cases} \right. \\
& \varepsilon_{\tilde{z}}(x)= \begin{cases}\varepsilon_{\widetilde{z l}}(x) & p^{3} \leq x \leq q^{3} \\
\varphi & q^{3} \leq x \leq r^{3} \\
\varepsilon_{\widetilde{z u}}(x) & r^{3} \leq x \leq s^{3} \\
1 & \text { otherwise }\end{cases}
\end{aligned}
$$

\section{Linear Generalized Neutrosophic Number}

In this section, we introduce the linear and generalized neutrosophic number. Previously the researcher take the maximum value of truth, indeterminacy and falsity function as 1 . But why it is a fixed value? for different decision makers view point any one can fixed the scale in a certain quantity which is less than one (but the quantity belong to zero to one). The we make a generalized definition of neutrosophic number for all type of decision makers. In this viewpoint we construct the generalized neutrosophic number. 


\subsection{Trapezoidal Single Valued Neutrosophic Number of category 1: When the quantity of the truth, indeterminacy and falsity are not dependent}

This case arises for a problem like "Selection of the member X in an election". Some people will surely vote for X, that is the truth function. Some of them will surely give their vote against X, which is the falsity function. Some of them will hesitate to cast their vote. Here, the entire component is distinct and not dependent.

A Trapezoidal Single Valued Neutrosophic Number of category 1 is defined as $\tilde{A}_{T N e}=\left(p_{1}, p_{2}, p_{3}, p_{4} ; q_{1}\right.$, $\left.q_{2}, q_{3}, q_{4} ; r_{1}, r_{2}, r_{3}, r_{4}\right)$, whose truth membership; indeterminacy membership and falsity membership are defined as follows:

$$
\begin{gathered}
T_{\tilde{A}_{T N e}}(x)= \begin{cases}\frac{x-p_{1}}{p_{2}-p_{1}} & \text { when } p_{1} \leq x<p_{2} \\
1 & \text { when } p_{2}<x \leq p_{3} \\
\frac{p_{4}-x}{p_{4}-p_{3}} & \text { when } p_{3}<x \leq p_{4} \\
0 & \text { otherwise }\end{cases} \\
I_{\tilde{A}_{T N e}}(x)= \begin{cases}\frac{q_{2}-x}{q_{2}-q_{1}} & \text { when } q_{1} \leq x<q_{2} \\
0 & \text { when } q_{3}<x \leq q_{4} \\
\frac{x-q_{3}}{q_{4}-q_{3}} & \text { when } q_{3}<x \leq q_{4} \\
1 & \text { otherwise }\end{cases} \\
F_{\tilde{A}_{T N e}}(x)= \begin{cases}\frac{r_{2}-x}{r_{2}-r_{1}} & \text { when } r_{1} \leq x<r_{2} \\
0 & \text { when } r_{2}<x \leq r_{3} \\
\frac{x-r_{3}}{r_{4}-r_{3}} & \text { when } r_{3}<x \leq r_{4} \\
1 & \text { otherwise }\end{cases}
\end{gathered}
$$

where $-0 \leq T_{\tilde{A}_{T N e}}(x)+I_{\tilde{A}_{T N e}}(x)+F_{\tilde{A}_{T N e}}(x) \leq 3+, x \in \tilde{A}_{T N e}$.

The parametric form of the above type number is

$$
\left(\tilde{A}_{T N e}\right)_{\mu, \vartheta, \varphi}=\left[T_{T N e 1}(\mu), T_{T N e 2}(\mu) ; I_{T N e 1}(\vartheta), I_{T N e 2}(\vartheta) ; F_{T N e 1}(\varphi), F_{T N e 2}(\varphi)\right]
$$

where,

$$
\begin{aligned}
T_{T N e 1}(\mu) & =p_{1}+\mu\left(p_{2}-p_{1}\right) \\
T_{T N e 2}(\mu) & =p_{4}-\mu\left(p_{4}-p_{3}\right) \\
I_{T N e 1}(\vartheta) & =q_{2}-\vartheta\left(q_{2}-q_{1}\right) \\
I_{T N e 2}(\vartheta) & =q_{3}+\vartheta\left(q_{4}-q_{3}\right) \\
F_{T N e 1}(\varphi) & =r_{2}-\varphi\left(r_{2}-r_{1}\right) \\
F_{T N e 2}(\varphi) & =r_{3}+\varphi\left(r_{4}-r_{3}\right) .
\end{aligned}
$$

Here, $0<\mu \leq 1,0<\vartheta \leq 1,0<\varphi \leq 1$ and $-0<\mu+\vartheta+\varphi \leq 3+$.

Note 3.1. In paper [15] the author represent the single valued trapezoidal neutrosophic number by four components $(a, b, c$ and $d)$ and three membership value of truthness, indeterminacy and falseness $\left(w_{\tilde{a}}, u_{\tilde{a}}\right.$ and $y_{\tilde{a}}$ which are belongs to $\left.[0,1]\right)$. But, in our approach we take sixteen different components $\left(p_{1}, p_{2}, p_{3}, p_{4} ; q_{1}, q_{2}, q_{3}, q_{4} ; r_{1}, r_{2}, r_{3}, r_{4}\right)$. That means both the definition are same, but we consider the asymmetric view of three membership functions individually. If anyone consider $p_{1}=q_{1}=r_{1}=a ; p_{2}=q_{2}=r_{2}=$ $b ; p_{3}=q_{3}=r_{3}=c ; p_{4}=q_{4}=r_{4}=d$ and take the maximum value of truthness, indeterminacy and falseness membership function as generalized value $w_{\tilde{a}}, u_{\tilde{a}}, y_{\tilde{a}}$ rather than 1 respectively then the definition in trapezoidal neutrosophic number in [15] comes. 


\subsection{Trapezoidal Single Valued Neutrosophic Number of category 2: When the quantity of indeterminacy and falsity are dependent}

This case may arise for a problem like "Selection of the member X in an election". Some people will surely vote for $\mathrm{X}$, that is the truth function. Some of them will not like to give their vote to X, but they hesitate to cast their vote to the other members. Here, the component of hesitant and falsity section are dependent.

A Trapezoidal Single Valued Neutrosophic Number (TrSVNNs) of category 2 is defined as $\tilde{A}_{T N e}=$ $\left(p_{1}, p_{2}, p_{3}, p_{4} ; q_{1}, q_{2}, q_{3}, q_{4} ; u_{N e}, y_{N e}\right)$ whose truth membership; indeterminacy membership and falsity membership are defined as follows:

$$
\begin{gathered}
T_{\tilde{A}_{T N e}}(x)= \begin{cases}\frac{x-p_{1}}{p_{2}-p_{1}} & \text { when } p_{1} \leq x<p_{2} \\
1 & \text { when } p_{2}<x \leq p_{3} \\
\frac{p_{4}-x}{p_{4}-p_{3}} & \text { when } p_{3}<x \leq p_{4} \\
0 & \text { otherwise }\end{cases} \\
I_{\tilde{A}_{T N e}}(x)= \begin{cases}\frac{q_{2}-x+u_{N e}\left(x-q_{1}\right)}{q_{2}-q_{1}} & \text { when } q_{1} \leq x<q_{2} \\
u_{N e} & \text { when } q_{2}<x \leq q_{3} \\
\frac{x-q_{3}+u_{N e}\left(q_{4}-x\right)}{q_{4}-q_{3}} & \text { when } q_{3}<x \leq q_{4} \\
1 & \text { otherwise }\end{cases}
\end{gathered}
$$

and

$$
F_{\tilde{A}_{T N e}}(x)= \begin{cases}\frac{q_{2}-x+y_{N e}\left(x-q_{1}\right)}{q_{2}-q_{1}} & \text { when } q_{1} \leq x<q_{2} \\ y_{N e} & \text { when } q_{2}<x \leq q_{3} \\ \frac{x-q_{2}+y_{N e}\left(q_{3}-x\right)}{q_{3}-q_{2}} & \text { when } q_{3}<x \leq q_{4} \\ 1 & \text { otherwise }\end{cases}
$$

where, $-0 \leq T_{\tilde{A}_{T N e}}(x)+I_{\tilde{A}_{T N e}}(x)+F_{\tilde{A}_{T N e}}(x) \leq 2+, x \in \tilde{A}_{T N e}$.

The parametric form of the above type number is

$$
\left(\tilde{A}_{T N e}\right)_{\mu, \vartheta, \gamma}=\left[T_{T N e 1}(\mu), T_{T N e 2}(\mu) ; I_{T N e 1}(\vartheta), I_{T N e 2}(\vartheta) ; F_{T N e 1}(\varphi), F_{T N e 2}(\varphi)\right]
$$

where

$$
\begin{aligned}
& T_{T N e 1}(\mu)=p_{1}+\mu\left(p_{2}-p_{1}\right) \\
& T_{T N e 2}(\mu)=p_{4}-\mu\left(p_{4}-p_{3}\right) \\
& I_{T N e 1}(\vartheta)=\frac{q_{2}-u_{N e} q_{1}-\vartheta\left(q_{2}-q_{1}\right)}{1-u_{N e}} \\
& I_{T N e 2}(\vartheta)=\frac{q_{3}-u_{N e} q_{4}+\vartheta\left(q_{4}-q_{3}\right)}{1-u_{N e}} \\
& F_{T N e 1}(\varphi)=\frac{q_{2}-y_{N e} q_{1}-\varphi\left(q_{2}-q_{1}\right)}{1-y_{N e}} \\
& F_{T N e 2}(\varphi)=\frac{q_{3}-y_{N e} q_{4}+\varphi\left(q_{4}-q_{3}\right)}{1-y_{N e}} .
\end{aligned}
$$

Here, $0<\mu \leq 1, u_{N e}<\vartheta \leq 1, y_{N e}<\varphi \leq 1$ and $-0<\vartheta+\varphi \leq 1+$ and $-0<\mu+\vartheta+\varphi \leq 2+$.

\subsection{Trapezoidal Single Valued Neutrosophic Number of category 3: When the quantity of the truth, indeterminacy and falsity are dependent}

This case may arises for a problem like suppose an author created one research paper but he/she don't know whether it will be accepted in a journal or not (hesitant function). After submission process reviewers can accept it (truth function) or can reject it (falsity). In this section all the three components are dependent. 
A TrSVNNs of category 3 is defined as $\tilde{A}_{T N e}=\left(p_{1}, p_{2}, p_{3}, p_{4} ; w_{N e}, u_{N e}, y_{N e}\right)$ whose truth membership; indeterminacy membership and falsity membership are defined as follows:

$$
\begin{gathered}
T_{\tilde{A}_{T N e}}(x)= \begin{cases}w_{N e \frac{x-p_{1}}{p_{2}-p_{1}}} & \text { when } p_{1} \leq x<p_{2} \\
w_{N e} & \text { when } p_{2}<x \leq p_{3} \\
w_{N e \frac{p_{4}-x}{p_{4}-p_{3}}} & \text { when } p_{3}<x \leq p_{4} \\
0 & \text { otherwise }\end{cases} \\
I_{\tilde{A}_{T N e}}(x)= \begin{cases}\frac{p_{2}-x+u_{N e}\left(x-p_{1}\right)}{p_{2}-p_{1}} & \text { when } p_{1} \leq x<p_{2} \\
u_{N e} & \text { when } p_{2}<x \leq p_{3} \\
\frac{x-p_{3}+u_{N e}\left(p_{4}-x\right)}{p_{4}-p_{3}} & \text { when } p_{3}<x \leq p_{4} \\
1 & \text { otherwise }\end{cases}
\end{gathered}
$$

and

$$
F_{\tilde{A}_{T N e}}(x)= \begin{cases}\frac{p_{2}-x+y_{N e}\left(x-p_{1}\right)}{p_{2}-p_{1}} & \text { when } p_{1} \leq x<p_{2} \\ y_{N e} & \text { when } p_{2}<x \leq p_{3} \\ \frac{x-p_{3}+y_{N e}\left(p_{4}-x\right)}{p_{4}-p_{3}} & \text { when } p_{3}<x \leq p_{4} \\ 1 & \text { otherwise }\end{cases}
$$

where $-0 \leq T_{\tilde{A}_{T N e}}(x)+I_{\tilde{A}_{T N e}}(x)+F_{\tilde{A}_{T N e}}(x) \leq 1+, x \in \tilde{A}_{T N e}$.

The parametric form of the above type number is

$$
\left(\tilde{A}_{N e}\right)_{\mu, \vartheta, \gamma}=\left[T_{T N e 1}(\mu), T_{T N e 2}(\mu) ; I_{T N e 1}(\vartheta), I_{T N e 2}(\vartheta) ; F_{T N e 1}(\varphi), F_{T N e 2}(\varphi)\right]
$$

where

$$
\begin{aligned}
T_{T N e 1}(\mu) & =p_{1}+\frac{\mu}{w_{N e}}\left(p_{2}-p_{1}\right) \\
T_{T N e 2}(\mu) & =p_{4}-\frac{\mu}{w_{N e}}\left(p_{4}-p_{3}\right) \\
I_{T N e 1}(\vartheta) & =\frac{p_{2}-u_{N e} p_{1}-\vartheta\left(p_{2}-p_{1}\right)}{1-u_{N e}} \\
I_{T N e 2}(\vartheta) & =\frac{p_{3}-u_{N e} p_{4}+\vartheta\left(p_{4}-p_{3}\right)}{1-u_{N e}} \\
F_{T N e 1}(\varphi) & =\frac{p_{2}-y_{N e} p_{1}-\varphi\left(p_{2}-p_{1}\right)}{1-y_{N e}} \\
F_{T N e 2}(\varphi) & =\frac{p_{3}-y_{N e} p_{4}+\varphi\left(p_{4}-p_{3}\right)}{1-y_{N e}} .
\end{aligned}
$$

Here, $0<\mu \leq w_{N e}, u_{N e}<\vartheta \leq 1, y_{N e}<\varphi \leq 1$ and $-0<\mu+\vartheta+\varphi \leq 1+$.

\section{Non-linear Generalized Neutrosophic Number}

The previous section we construct the generalized neutrosophic number. Now when we take the truth, indeterminacy and falsity functions and think about the pictorial representation then we see that the borderline is always a straight line due to the linearity of the define function. Now we can make the function generalized. We can give a degree of the function as per uncertainty. Which is the basic concept of non-linear neutrosophic number. 


\subsection{Single-valued Non Linear Trapezoidal Neutrosophic Number with twelve components}

A single-valued Non Linear Trapezoidal Neutrosophic Number with twelve components is defined as $\tilde{A}_{T N e}=$ $\left(a_{1}, a_{2}, a_{3}, a_{4} ; b_{1}, b_{2}, b_{3}, b_{4} ; c_{1}, c_{2}, c_{3}, c_{4}\right)$ whose truth membership, indeterminacy membership and falsity membership are defined as:

$$
\begin{array}{r}
T_{\tilde{A}_{T N e}}(x)= \begin{cases}\left(\frac{x-a_{1}}{a_{2}-a_{1}}\right)^{p_{1}} & \text { when } a_{1} \leq x<a_{2} \\
1 & \text { when } a_{2}<x \leq a_{3} \\
\left(\frac{a_{3}-x}{a_{3}-a_{2}}\right)^{p_{2}} & \text { when } a_{3}<x \leq a_{4} \\
0 & \text { otherwise }\end{cases} \\
I_{\tilde{A}_{T N e}}(x)= \begin{cases}\left(\frac{x-b_{1}}{b_{2}-b_{1}}\right)^{q_{1}} & \text { when } b_{1} \leq x<b_{2} \\
0 & \text { when } b_{2}<x \leq b_{3} \\
\left(\frac{x-b_{3}}{b_{3}-b_{2}}\right)^{q_{2}} & \text { when } b_{3}<x \leq b_{4} \\
1 & \text { otherwise }\end{cases}
\end{array}
$$

and

$$
F_{\tilde{A}_{T N e}}(x)= \begin{cases}\left(\frac{x-c_{1}}{c_{2}-c_{1}}\right)^{r_{1}} & \text { when } c_{1} \leq x<c_{2} \\ 0 & \text { when } c_{2}<x \leq c_{3} \\ \left(\frac{x-c_{3}}{c_{3}-c_{2}}\right)^{r_{2}} & \text { when } c_{3}<x \leq c_{4} \\ 1 & \text { otherwise }\end{cases}
$$

where, $0 \leq T_{\tilde{A}_{T N e}}(x)+I_{\tilde{A}_{T N e}}(x)+F_{\tilde{A}_{T N e}}(x) \leq 3, x \in \tilde{A}_{T N e} \& p_{1}, p_{2}, q_{1}, q_{2}, r_{1}, r_{2}>$ or, $<1$ define the non linearity. If $p_{1}, p_{2}, q_{1}, q_{2}, r_{1}, r_{2}=1$ then it becomes linear neutrosophic number with twelve component.

\subsection{Single-valued Generalized Trapezoidal Neutrosophic Number with twelve components}

A single-valued Trapezoidal Neutrosophic number with twelve components is defined as $\tilde{A}_{T N e}=$ $\left(a_{1}, a_{2}, a_{3}, a_{4} ; b_{1}, b_{2}, b_{3}, b_{4} ; c_{1}, c_{2}, c_{3}, c_{4}\right)$, whose truth membership, indeterminacy membership and falsity membership are defined as:

$$
\begin{gathered}
T_{\tilde{A}_{T N e}}(x)= \begin{cases}\omega \frac{x-a_{1}}{a_{2}-a_{1}} & \text { when } a_{1} \leq x<a_{2} \\
\omega \frac{a_{4}-x}{a_{4}-a_{3}} & \text { when } a_{2}<x \leq a_{3} \\
0 & \text { otherwise }\end{cases} \\
I_{\tilde{A}_{T N e}}(x)= \begin{cases}\rho \frac{x-b_{1}}{b_{2}-b_{1}} & \text { when } b_{1} \leq x<b_{2} \\
0 & \text { when } b_{2}<x \leq b_{3} \\
\rho \frac{x-b_{4}}{b_{4}-b_{3}} & \text { when } b_{3}<x \leq b_{4} \\
\rho & \text { otherwise }\end{cases}
\end{gathered}
$$

and

$$
F_{\tilde{A}_{T N e}}(x)= \begin{cases}\lambda \frac{x-c_{1}}{c_{2}-c_{1}} & \text { when } c_{1} \leq x<c_{2} \\ 0 & \text { when } c_{2}<x \leq c_{3} \\ \lambda \frac{x-c_{4}}{c_{4}-c_{3}} & \text { when } c_{3}<x \leq c_{4} \\ \lambda & \text { otherwise }\end{cases}
$$

where $0 \leq T_{\tilde{A}_{T N e}}(x)+I_{\tilde{A}_{T N e}}(x)+F_{\tilde{A}_{T N e}}(x) \leq 3, x \in \tilde{A}_{T N e}$. 
The parametric form of the above type number is

$$
\left(\tilde{A}_{T N e}\right)_{\alpha, \beta, \gamma}=\left[T_{T N e 1}(\alpha), T_{T N e 2}(\alpha) ; I_{T N e 1}(\beta), I_{T N e 2}(\beta) ; F_{T N e 1}(\gamma), F_{T N e 2}(\gamma)\right]
$$

where,

$$
\begin{aligned}
& T_{T N e 1}(\alpha)=a_{1}+\alpha \frac{\left(a_{2}-a_{1}\right)}{\omega} \\
& T_{T N e 2}(\alpha)=a_{4}-\alpha \frac{\left(a_{4}-a_{3}\right)}{\omega} \\
& I_{T N e 1}(\beta)=b_{2}-\beta \frac{\left(b_{2}-b_{1}\right)}{\rho} \\
& I_{T N e 2}(\beta)=b_{3}+\beta \frac{\left(b_{4}-b_{3}\right)}{\rho} \\
& F_{T N e 1}(\gamma)=c_{2}-\gamma \frac{\left(c_{2}-c_{1}\right)}{\lambda} \\
& F_{T N e 2}(\gamma)=c_{3}+\gamma \frac{\left(c_{4}-c_{3}\right)}{\lambda} .
\end{aligned}
$$

Here, $0<\alpha \leq 1,0<\beta \leq 1,0<\gamma \leq 1$ and $-0<\alpha+\beta+\gamma \leq 3+$.

\subsection{Single-valued Generalized Non Linear Trapezoidal Neutrosophic Number with twelve components}

A single-valued Non Linear Trapezoidal Neutrosophic number with twelve components is defined as $\tilde{A}_{T N e}=$ $\left(a_{1}, a_{2}, a_{3}, a_{4} ; b_{1}, b_{2}, b_{3}, b_{4} ; c_{1}, c_{2}, c_{3}, c_{4}\right)$ whose truth membership, indeterminacy membership and falsity membership are defined as

$$
\begin{gathered}
T_{\tilde{A}_{T N e}}(x)= \begin{cases}\omega\left(\frac{x-a_{1}}{a_{2}-a_{1}}\right)^{p_{1}} & \text { when } a_{1} \leq x<a_{2} \\
\omega\left(\frac{a_{3}-x}{a_{3}-a_{2}}\right)^{p_{2}} & \text { when } a_{2}<x \leq a_{3} \\
0 & \text { when } a_{3}<x \leq a_{4}\end{cases} \\
I_{\tilde{A}_{T N e}}(x)= \begin{cases}\rho\left(\frac{x-b_{1}}{b_{2}-b_{1}}\right)^{q_{1}} & \text { wherwise } b_{1} \leq x<b_{2} \\
0 & \text { when } b_{2}<x \leq b_{3} \\
\rho\left(\frac{x-b_{3}}{b_{3}-b_{2}}\right)^{q_{2}} & \text { when } b_{3}<x \leq b_{4} \\
\rho & \text { otherwise }\end{cases}
\end{gathered}
$$

and

$$
F_{\tilde{A}_{T N e}}(x)= \begin{cases}\lambda\left(\frac{x-c_{1}}{c_{2}-c_{1}}\right)^{r_{1}} & \text { when } c_{1} \leq x<c_{2} \\ 0 & \text { when } c_{2}<x \leq c_{3} \\ \lambda\left(\frac{x-c_{3}}{c_{3}-c_{2}}\right)^{r_{2}} & \text { when } c_{3}<x \leq c_{4} \\ \lambda & \text { otherwise }\end{cases}
$$

where, $-0 \leq T_{\tilde{A}_{T N e}}(x)+I_{\tilde{A}_{T N e}}(x)+F_{\tilde{A}_{T N e}}(x) \leq 3+, x \in \tilde{A}_{T N e}$.

\section{Defuzzification of a Linear Trapezoidal Neutrosophic Number}

Defuzzification is the process of generating a perceptible result in crisp logic corresponding to the fuzzy number and its membership function. Researchers all around the universe are interested in this fact that if 


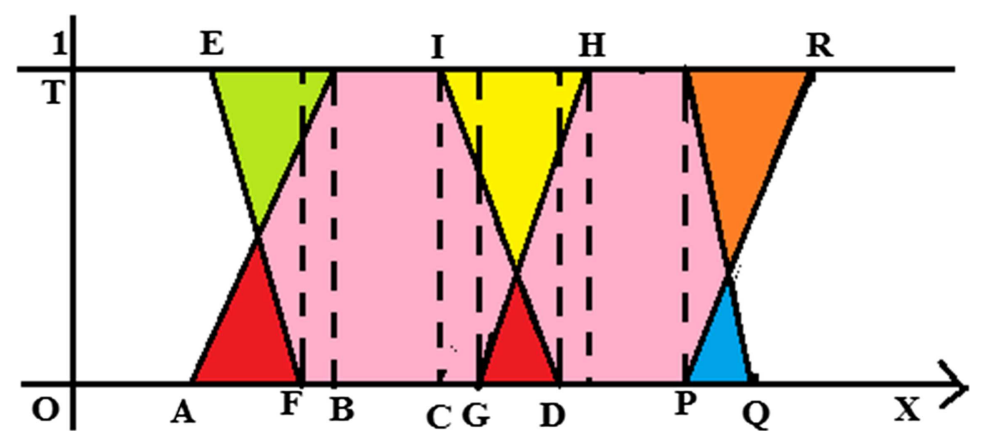

FIgURE 1. Drawing some trapezium from neutrosophic trapezoidal fuzzy number.

there is a fuzzy number with its membership function, then what will be the crisp number related with the fuzzy number? They proposed some useful methods to convert a fuzzy number into a crisp number. Some well known methods are BADD (basic defuzzification distributions), BOA (bisector of area), CDD (constraint decision defuzzification), COA (center of area), COG (center of gravity), ECOA (extended center of area), EQM (extended quality method), FCD (fuzzy clustering defuzzification) etc. This process actually maps a fuzzy number into a crisp number. It is widely used in fuzzy control system also.

In our neutrosophic environment researchers are very much interested to convert a neutrosophic number into a crisp number. In case of neutrosophic fuzzy number where there are three different kind of membership functions are present we proposed two methods

(1) Removal area method and

(2) Mean interval method

to convert a trapezoidal neutrosophic fuzzy number into a crisp number.

\subsection{Defuzzification using Removal Area Method:}

Let us consider a linear trapezoidal neutrosophic fuzzy number as follows (Fig. 1):

Let us consider a real number $r \in R$. We also consider a fuzzy number $\check{M}$ associated with the lower trapezium, and then left hand side removal area of $\check{M}$ with respect to $r$ is $R_{l}(\check{M}, r)$, and is defined as the area bounded by $r$ and the left hand side of the fuzzy number $\check{M}$. Similarly, if we consider the right hand side removal of $\check{M}$ with respect to $r$ it will be $R_{r}(\check{M}, r)$; we also consider a real number $r \in R$ and a fuzzy number $\check{N}$ associated with the left most upper trapezium ( $\mathbf{E F G H})$, then left hand side removal area of $\check{N}$ with respect to $r$ will be $R_{l}(\check{N}, r)$, and is defined as the area bounded by $r$ and the left side of the fuzzy number $\check{N}$. Similarly, if we consider the right hand side removal area of $\check{N}$ with respect to $r$ it will be $R_{r}(\check{N}, r)$, A fuzzy number $\check{P}$ associated with the right most upper trapezium (IDPR), then left side removal of $\check{P}$ with respect to $r$ is $R_{l}(\check{P}, r)$, and is defined as the area bounded by $r$ and the left hand side of the fuzzy number $\check{P}$. Finally, the right hand side removal of $\check{P}$ with respect to $r$ is $R_{r}(\check{P}, r)$.

Mean is defined as

$$
\begin{aligned}
R(\check{M}, r) & =\frac{R_{l}(\check{M}, r)+R_{r}(\check{M}, r)}{2}, \\
R(\check{N}, r) & =\frac{R_{l}(\check{N}, r)+R_{r}(\check{N}, r)}{2} \\
R(\check{P}, r) & =\frac{R_{l}(\check{P}, r)+R_{r}(\check{P}, r)}{2} .
\end{aligned}
$$


Then, we define the defuzzification of a linear neutrosophic trapezoidal fuzzy as:

$$
R(\check{D}, r)=\frac{R(\check{M}, r)+R(\check{N}, r)+R(\check{P}, r)}{3} .
$$

For $r=0$,

$$
R(\check{M}, 0)=\frac{R_{l}(\check{M}, 0)+R_{r}(\check{M}, 0)}{2}, \quad R(\check{N}, 0)=\frac{R_{l}(\check{N}, 0)+R_{r}(\check{N}, 0)}{2}, \quad R(\check{P}, 0)=\frac{R_{l}(\check{P}, 0)+R_{r}(\check{P}, 0)}{2} .
$$

Then,

$$
(\check{D}, 0)=\frac{R(\check{M}, 0)+R(\check{N}, 0)+R(\check{P}, 0)}{3} .
$$

We take $\check{A}=\left(a_{1}, b_{1}, c_{1}, d_{1}\right), \check{B}=\left(e_{1}, f_{1}, g_{1}, h_{1}\right), \check{C}=\left(i_{1}, j_{1}, k_{1}, l_{1}\right)$ (Fig. 2).

Here, Red marked portions denoted the corresponding area of the truth membership function, Green marked portions denoted the corresponding area of the falsity membership function and saffron marked portions denoted the corresponding area of the hesitation membership function.

Then,

$$
\begin{aligned}
& R_{l}(\check{M}, 0)=\text { area of trapezium OABT }=\frac{\left(a_{1}+b_{1}\right)}{2} \cdot 1 \\
& R_{r}(\check{M}, 0)=\text { area of trapezium OQPT }=\frac{\left(c_{1}+d_{1}\right)}{2} \cdot 1 \\
& R_{l}(\check{N}, 0)=\text { area of trapezium OFET }=\frac{\left(e_{1}+f_{1}\right)}{2} \cdot 1 \\
& R_{r}(\check{N}, 0)=\text { area of trapezium OGHT }=\frac{\left(g_{1}+h_{1}\right)}{2} \cdot 1 \\
& R_{l}(\check{P}, 0)=\text { area of trapezium ODIT }=\frac{\left(i_{1}+j_{1}\right)}{2} \cdot 1 \\
& R_{r}(\check{P}, 0)=\text { area of trapezium OPRT }=\frac{\left(k_{1}+l_{1}\right)}{2} \cdot 1 .
\end{aligned}
$$

Hence,

$$
R(\check{M}, 0)=\frac{\left(a_{1}+b_{1}+c_{1}+d_{1}\right)}{4}, \quad R(\check{N}, 0)=\frac{\left(e_{1}+f_{1}+g_{1}+h_{1}\right)}{4}, \quad R(\check{P}, 0)=\frac{\left(i_{1}+j_{1}+k_{1}+l_{1}\right)}{4} .
$$

So,

$$
R(\check{D}, 0)=\frac{\left(a_{1}+b_{1}+c_{1}+d_{1}+e_{1}+f_{1}+g_{1}+h_{1}+i_{1}+j_{1}+k_{1}+l_{1}\right)}{12} .
$$

\subsection{De-neutrosophication technique using mean interval method}

From 3.1 we can write the parametric form of the trapezoidal neutrosophic number is as follows,

$$
\begin{aligned}
& T_{T N e 1}(\mu)=p_{1}+\mu\left(p_{2}-p_{1}\right) \\
& T_{T N e 2}(\mu)=p_{4}-\mu\left(p_{4}-p_{3}\right) \\
& I_{T N e 1}(\vartheta)=q_{2}-\vartheta\left(q_{2}-q_{1}\right) \\
& I_{T N e 2}(\vartheta)=q_{3}+\vartheta\left(q_{4}-q_{3}\right) \\
& F_{T N e 1}(\varphi)=r_{2}-\varphi\left(r_{2}-r_{1}\right) \\
& F_{T N e 2}(\varphi)=r_{3}+\varphi\left(r_{4}-r_{3}\right) .
\end{aligned}
$$



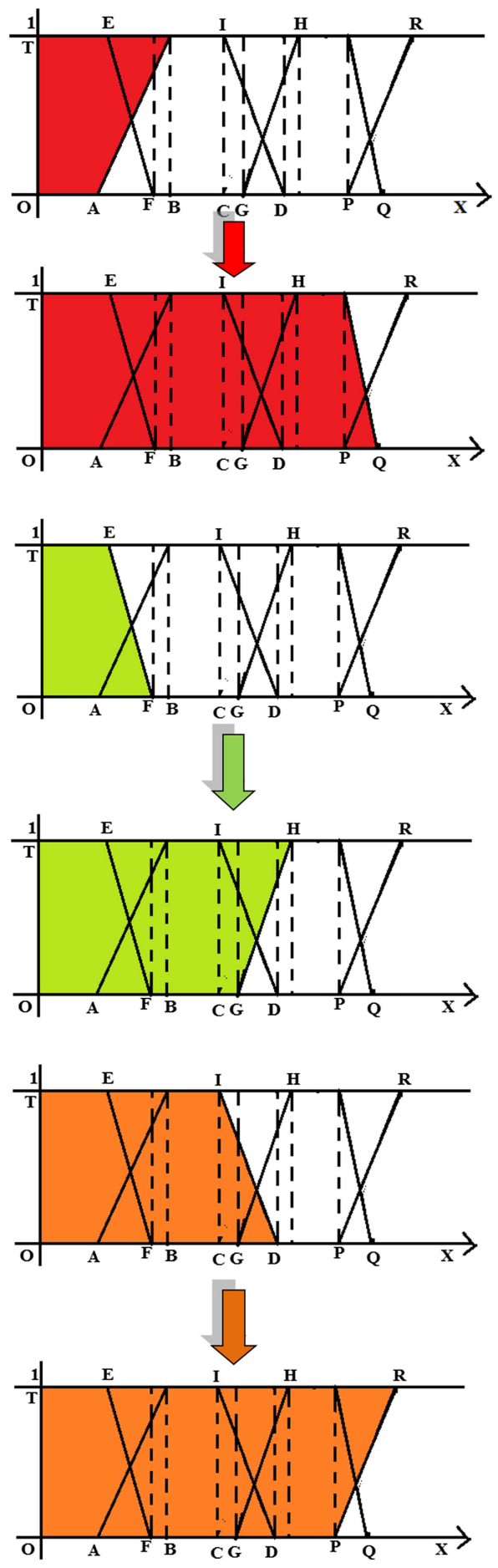

Figure 2. De-Neutrosophication techniques using removal area method. 
TABLE 1. Different trapezoidal neutrosophic number with de-neutrosophication value, hamming distance and euclidean distance.

\begin{tabular}{|c|c|c|c|c|c|}
\hline No. of set & $\begin{array}{l}\text { Trapezoidal neutro- } \\
\text { sophic number }\end{array}$ & $\begin{array}{l}\text { De- } \\
\text { neutrosophication } \\
\text { value }\end{array}$ & $\begin{array}{l}\text { Centroid point by } \\
\text { Deli's approach [13] }\end{array}$ & $\begin{array}{l}\text { Hamming } \\
\text { distance [13] }\end{array}$ & $\begin{array}{l}\text { Euclidean } \\
\text { distance [13] }\end{array}$ \\
\hline Set 1 & $\begin{array}{l}(1,2,3,4 ; \\
0.5,1.5,2.5,3.5 ; \\
1.2,2.7,3.5,4.5 \mid \\
0.9,0.8,0.8)\end{array}$ & 2.4917 & $\begin{array}{l}{[(0.33,0.36) ;} \\
(9.46,0.92) ; \\
(9.46,0.92)]\end{array}$ & 17.11 & 12.08 \\
\hline Set 2 & $\begin{array}{l}(0.5,1.5,2.5,3.5 \\
0.3,1.3,2.2,3.2 ; \\
0.7,1.7,2.2,3.8 \mid \\
0.9 ; 0.8 ; 0.8)\end{array}$ & 1.95 & $\begin{array}{l}{[(0.52,0.32) ;} \\
(8.36,0.92) ; \\
(8.36,0.92)]\end{array}$ & 15.08 & 10.52 \\
\hline Set 3 & $\begin{array}{l}(0.3,1.2,2.8,3.8 \\
0.5,1.5,2.5,3.5 ; \\
0.8,1.7,2.7,3.8 \mid \\
0.9 ; 0.8 ; 0.8)\end{array}$ & 2.0917 & $\begin{array}{l}{[(0.32,0.38) ;} \\
(3.74,0.92) ; \\
((3.74,0.92)]\end{array}$ & 5.7 & 3.99 \\
\hline Set 4 & $\begin{array}{l}(1,3,5,7 ; \\
0.5,1.5,2.5,3.5 ; \\
1.2,2.7,4.5,7.5 \mid \\
0.9 ; 0.8 ; 0.8)\end{array}$ & 3.325 & $\begin{array}{l}{[(1.4,0.39) ;} \\
(11.12,0.91) ; \\
(11.12,0.91)]\end{array}$ & 21.43 & 14.47 \\
\hline
\end{tabular}

TABLE 2. Comparison table of ranking.

\begin{tabular}{lll}
\hline \hline & Methods & Ranking \\
\hline 1 & By Our De-Neutrosophication Value Concept & Set $4>$ Set $1>$ Set $3>$ Set 2 \\
2 & By Deli's Hamming distance approach [13] & Set $4>$ Set $1>$ Set $2>$ Set 3 \\
3 & By Deli's Euclidean distance approach [13] & Set $4>$ Set $1>$ Set $2>$ Set 3 \\
\hline
\end{tabular}

Here, $0<\mu \leq 1,0<\vartheta \leq 1,0<\varphi \leq 1$ and $0<\mu+\vartheta+\varphi \leq 3$.

Now, de-neutrosophication is defined as,

$$
\begin{aligned}
\check{A} & =\frac{\int_{\mu=0}^{1} \frac{\left\{T_{T N e 1}(\mu)+T_{T N e 2}(\mu)\right\}}{2} \mathrm{~d} \mu+\int_{\vartheta=0}^{1} \frac{\left\{T_{T N e 1}(\vartheta)+T_{T N e 2}(\vartheta)\right\}}{2} \mathrm{~d} \vartheta+\int_{\varphi=0}^{1} \frac{\left\{T_{T N e 1}(\varphi)+T_{T N e 2}(\varphi)\right\}}{2} \mathrm{~d} \varphi}{3} \\
& =\frac{\int_{\mu=0}^{1} \frac{\left\{p_{1}+\mu\left(p_{2}-p_{1}\right)+p_{4}-\mu\left(p_{4}-p_{3}\right)\right\}}{2} \mathrm{~d} \mu+\int_{\vartheta=0}^{1} \frac{\left\{q_{2}-\vartheta\left(q_{2}-q_{1}\right)+q_{3}+\vartheta\left(q_{4}-q_{3}\right)\right\}}{2} \mathrm{~d} \vartheta+\int_{\varphi=0}^{1} \frac{\left\{r_{2}-\varphi\left(r_{2}-r_{1}\right)+r_{3}+\varphi\left(r_{4}-r_{3}\right)\right\}}{2} \mathrm{~d} \varphi}{3} \\
& =\frac{p_{1}+p_{2}+p_{3}+p_{4}+q_{1}+q_{2}+q_{3}+q_{4}+r_{1}+r_{2}+r_{3}+r_{4}}{12} .
\end{aligned}
$$

\subsection{Numerical examples}

See Table 1.

\subsubsection{Ranking using different methods}

See Table 2.

\subsection{Comparison between Triangular and Trapezoidal shape Neutrosophic number}

Measurement of vagueness is a very important topic in uncertainty theory. It is involves in fuzzy sets but not in crisp set. We know that fuzzy number can be taken as triangular form or in trapezoidal form; although we 
still don't know which form can store more fuzzy information. In case of neutrosophic environment we defined triangular neutrosophic number [34] and trapezoidal neutrosophic number [41] but we can't measure which form gives us better result and store more information.

Here, using de-neutrosophication idea we will try to built up a comparison between triangular neutrosophic fuzzy number and trapezoidal neutrosophic fuzzy number.

Avishek et al. [8] introduced the idea of de-neutrosophication for triangular neutrosophic fuzzy number as $R\left(\check{D}_{\Delta}, 0\right)=\frac{\left(a_{1}+2 b_{1}+c_{1}+d_{1}+2 e_{1}+f_{1}+g_{1}+2 h_{1}+k_{1}\right)}{12}$. Also in this paper we introduced deneutrosophication of trapezoidal neutrosophic fuzzy number using removal area method as $\left(\check{D}_{\square}, 0\right)=$ $\frac{\left(a_{1}+b_{1}+c_{1}+d_{1}+e_{1}+f_{1}+g_{1}+h_{1}+i_{1}+j_{1}+k_{1}+l_{1}\right)}{12}$.

If we take truth part range as $[a, d]$, falsity part as $[e, h]$ and hesitant part as $[i, l]$ for both the forms, then

$$
\begin{aligned}
R(D, 0) & =R\left(\check{D}_{\square}, 0\right)-R\left(\check{D}_{\Delta}, 0\right), \text { where } R(D, 0) \in \mathbb{R} \\
& =\frac{(a+b+c+d+e+f+g+h+i+j+k+l)}{12}-\frac{(a+2 . p+d+e+2 . q+h+i+2 . r+l)}{12} \\
& =\frac{(b+c-2 p)+(f+g-2 q)+(j+k-2 r)}{12},
\end{aligned}
$$

where $b<p<c, f<q<g, j<r<k$.

If $R(D, 0)>0$, Then trapezoidal shape associated with more vagueness information.

If $R(D, 0)<0$, Then triangular shape associated with more vagueness information.

and $R(D, 0)=0$, Then equal contribution.

\subsubsection{Example}

\begin{tabular}{llll}
\hline Trapezoidal neutrosophic number & Triangular neutrosophic number & $R(D, 0)$ & Result \\
\hline$(3,6,9,12 ; 2,5,8,11 ; 6,11,13,15)$ & $(3,7,12 ; 2,6,11 ; 6,12,15)$ & Positive & Trapezoidal better \\
$(2,5,8,11 ; 1,4,7,10 ; 5,10,12,14)$ & $(2,6,11 ; 1,5,10 ; 5,11,14)$ & Positive & Trapezoidal better \\
$(3,6,9,12 ; 2,5,8,11 ; 6,11,13,15)$ & $(3,8,12 ; 2,7,11 ; 6,12,15)$ & Negative & Triangular better \\
$(3,6,10,12 ; 2,6,8,11 ; 6,11,13,15)$ & $(3,8,12 ; 2,7,11 ; 6,12,15)$ & Zero & Same \\
\hline
\end{tabular}

\section{Crash model: Time Cost optimization in neUtrosophic EnVironment}

Before going to the main problem we need to clarify some factors involving the time cost optimization problem.

Project cost. In reference to the cost factor in the current project scheduling, there must be a relationship presented between the two parameters namely that of cost \& time duration involved in that particular activity. The Direct cost \& the indirect cost constitutes the total cost of any project.

Direct cost. There exists a direct rapport or a direct reference with the cost depending upon various factors of production like the amount of resources that the individual is furnished with for the execution of individual activities like the inputs equipped to the person for the management of the project which is described as "manpower loading" \& the consumption of materials. It is to be notified that there is an increment in the direct cost if there is a reduction in the duration that an activity consumes for its completion.

Indirect cost. This type of cost is fully related with overhead expenditure like managerial jobs, indirect supplies etc. The indirect cost is generally calculated for a week, or for a month basis. The indirect cost gradually decreases when the activity period is diminishing. 
Coming up to the network diagram, it is a progressive tool used to recognize the activities whose duration in time should be decremented \& shortened up into a laconic flowchart so that the time involved in completing the project can be quick \& in the most economic manner to complacent the urgent needs for project completion. Time is the indispensable crucial factor for finishing up any successful project for the dissemination of economic prosperity \& cost efficiency. It is the only measure to abrogate uneconomic ideas for any project to make the project a glorious success carried on by the virtue of Network Diagram. Carrying out a project in the shortest time by reducing the time duration or by the addition of some extra endeavor is called "Crashing the Activity". The crash time stands as the paragon for the representation of the minimum activity time \& if there are constant attempts to further crash would result in raising the activity cost without any alteration in the time factor.

The activity cost corresponding to the crash time eaten up in the project is called CRASH COST which is the lowest negligible direct cost required to archive \& encrypt the crash performance time.We are amplified with the definition of Normal time which is the time duration required to perform an activity of the absolute minimum of the direct cost which is the equivalent of the normal cost required to fund the specified activity.

Now,

$$
\text { Cost Slope }=\frac{\text { Crash Cost }- \text { Normal Cost }}{\text { Normal time }- \text { Crash Time }}
$$

Now, in this section we consider the normal time and the crash time both are trapezoidal neutrosophic fuzzy number and in this neutrosophic environment we convert the trapezoidal neutrosophic fuzzy number into a crisp number using the de-neutrosophication technique

$$
R(\check{D}, 0)=\frac{\left(a_{1}+b_{1}+c_{1}+d_{1}+e_{1}+f_{1}+g_{1}+h_{1}+i_{1}+j_{1}+k_{1}+l_{1}\right)}{12} .
$$

\subsection{Time-cost optimization algorithm}

\section{Step 1.}

Using de-neutrosophication technique convert the trapezoidal netrosophic fuzzy normal and crash time into a crisp number.

\section{Step 2 .}

Network diagram construction using the information in the table.

\section{Step 3.}

Find out normal critical path and identify the critical activities.

\section{Step 4.}

Evaluate cost slope for each activities and rank them.

\section{Step 5.}

Calculate the total cost of the project and continue the process until all the critical activities are entirely crashed or no more crashing is achievable.

\section{Step 6.}

Calculate Optimum cost and project duration. 


\subsection{Flowchart for time cost optimization techniques}

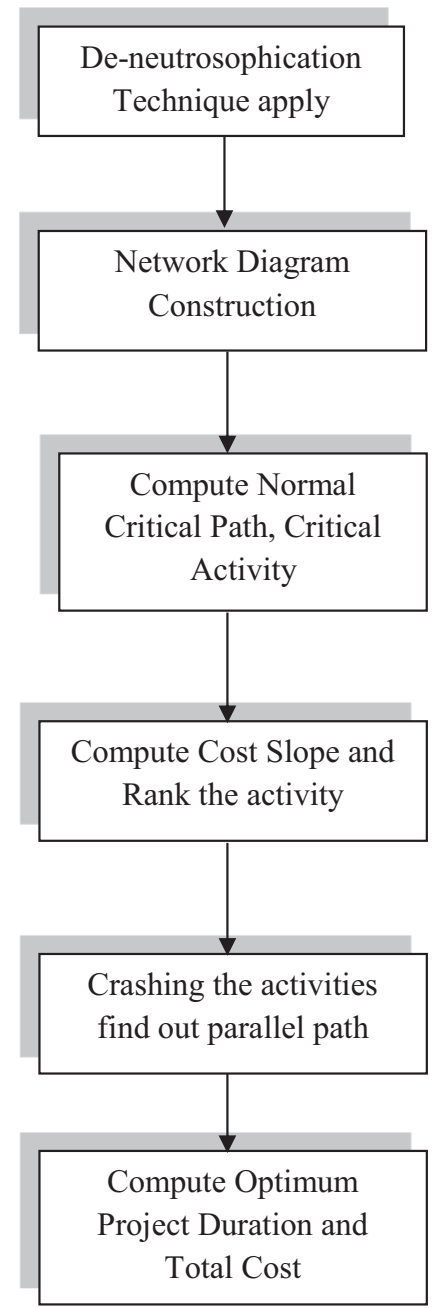

\subsection{Problem definition}

Let us consider a project for building up a testing facility. The list of activities with their time and cost data is given below

\begin{tabular}{|c|c|}
\hline Attribute/ Description & Normal and Crash time verbal phrase \\
\hline Design facility & $\begin{array}{l}\text { (Semi Medium, Medium, Quasi Medium, High; Low, Medium, Quasi Medium, } \\
\text { High; Medium, Quasi Medium, High, Very High) }\end{array}$ \\
\hline Receive equipment & $\begin{array}{l}\text { (Semi Medium, Medium, Quasi Medium, High; Low, Medium, Quasi Medium, } \\
\text { High; Medium, Quasi Medium, High, Very High) }\end{array}$ \\
\hline Selection of staff & $\begin{array}{l}\text { (Semi Medium, Medium, Quasi Medium, High; Low, Medium, Quasi Medium, } \\
\text { High; Medium, Quasi Medium, High, Very High) }\end{array}$ \\
\hline Training & $\begin{array}{l}\text { Semi Medium, Medium, Quasi Medium, High; Low, Medium, Quasi Medium, } \\
\text { High; Medium, Quasi Medium, High, Very High) }\end{array}$ \\
\hline Installation & $\begin{array}{l}\text { (Semi Medium, Medium, Quasi Medium, High; Low, Medium, Quasi Medium, } \\
\text { High; Medium, Quasi Medium, High, Very High) }\end{array}$ \\
\hline Test facilities & $\begin{array}{l}\text { (Semi Medium, Medium, Quasi Medium, High; Low, Medium, Quasi Medium, } \\
\text { High; Medium, Quasi Medium, High, Very High) }\end{array}$ \\
\hline
\end{tabular}




\begin{tabular}{|c|c|c|c|c|c|}
\hline \multirow[t]{2}{*}{ Activity } & \multirow[t]{2}{*}{ Description } & \multicolumn{2}{|l|}{ Normal } & \multicolumn{2}{|l|}{ Crash } \\
\hline & & Time & Cost & Time & Cost \\
\hline $1-2$ & Design facility & $\begin{array}{l}(3,6,9,12 \\
2.5,5,8,11.5 \\
5,8,11,15)\end{array}$ & 100 & $\begin{array}{l}(3.5,5,6.5,8 \\
3.25,4.5,6,7.75 \\
4.5,6,7.5,9.5)\end{array}$ & 200 \\
\hline $1-3$ & Receive equipment & $\begin{array}{l}(1.5,3,4.5,6 \\
1.25,2.5,4,5.75 \\
2.5,4,5.5,7.5)\end{array}$ & 150 & $\begin{array}{l}(0.75,1.5,2.25,3 ; \\
0.625,1.25,2,2.875 \\
1.25,2,2.75,3.75)\end{array}$ & 350 \\
\hline $2-4$ & Selection of staff & $\begin{array}{l}(0.75,1.5,2.25,3 ; \\
0.625,1.25,2,2.875 \\
1.25,2,2.75,3.75)\end{array}$ & 50 & $\begin{array}{l}(0.5,1,1.5,2 ; \\
0.25,0.5,0.75,1 ; \\
0.65,1.10,1.25,1.5)\end{array}$ & 90 \\
\hline $2-5$ & Training & $\begin{array}{l}(5,8,11,14 \\
4.5,7,10,13.5 \\
7,10,13,17)\end{array}$ & 100 & $\begin{array}{l}(2.5,4,5.5,7 \\
2.25,3.5,5,6.75 \\
3.5,5,6.5,8.5)\end{array}$ & 400 \\
\hline $3-4$ & Installation & $\begin{array}{l}(2.5,4,5.5,7 \\
2.25,3.5,5,6.75 \\
3.5,5,6.5,8.5)\end{array}$ & 100 & $\begin{array}{l}(0.4,1,1.5,2 ; \\
0.25,0.5,0.7,1.5 ; \\
0.45,1.05,1.20,1.445)\end{array}$ & 200 \\
\hline $4-5$ & Test facilities & $\begin{array}{l}(1.75,2.5,3.25,4 ; \\
1.625,2.25,3,3.875 \\
2.25,3,3.375,4.75)\end{array}$ & 80 & $\begin{array}{l}(0.5,1,1.5,2 ; \\
0.25,0.5,0.75,1 ; \\
0.65,1.10,1.25,1.5)\end{array}$ & 90 \\
\hline
\end{tabular}

Determine the optimum project duration and cost for the following data, given that indirect cost is Rs. 70 per day.

\section{Solution procedure}

The solution can be done by the following step as mention above. Here we only show the numerical study.

\section{Step 1.}

\begin{tabular}{llllll}
\hline \multirow{2}{*}{ Activity } & \multirow{2}{*}{ Description } & \multicolumn{2}{c}{ Normal } & \multicolumn{2}{c}{ Crash } \\
\cline { 3 - 6 } & & Time & Cost & Time & Cost \\
\hline $1-2$ & Design facility & 8 & 100 & 6 & 200 \\
$1-3$ & Receive equipment & 4 & 150 & 2 & 350 \\
$2-4$ & Selection of staff & 2 & 50 & 1 & 90 \\
$2-5$ & Training & 10 & 100 & 5 & 400 \\
$3-4$ & Installation & 5 & 100 & 1 & 200 \\
$4-5$ & Test facilities & 3 & 80 & 1 & 100 \\
\hline
\end{tabular}

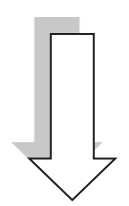

Step 2. Network construction. 


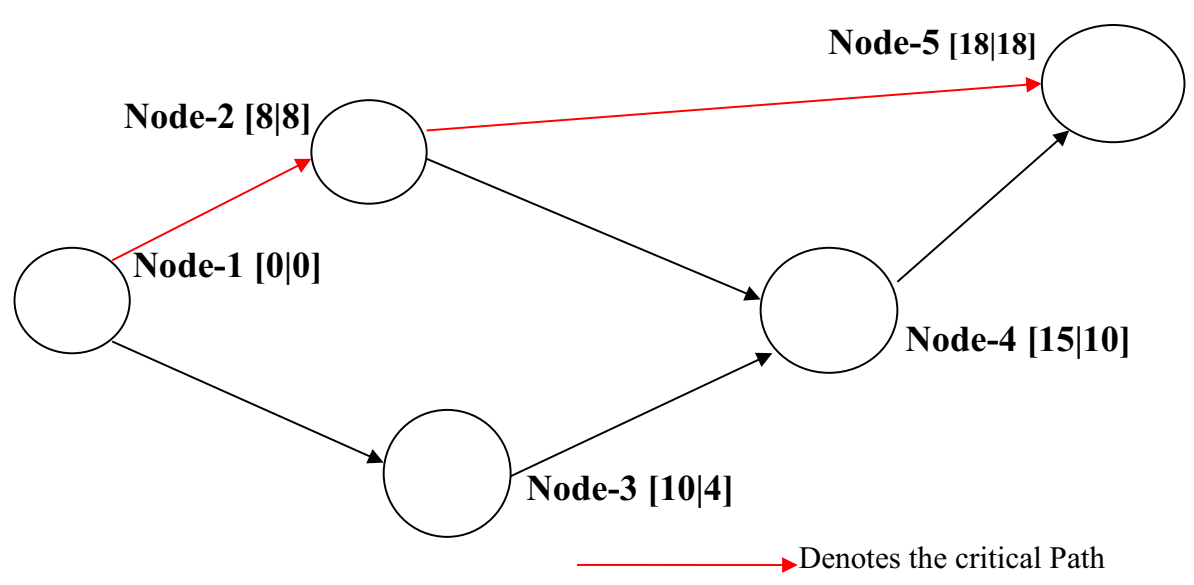

\section{Step 3.}

The required Critical Path $1 \rightarrow 2 \rightarrow 5$.

Project Normal Duration-18 days.

Cost of the project $=$ Normal cost + Indirect Cost $=$ Rs. 1840 .

\section{Step 4.}

The cost slope and the number of days to be crashed are given below

\begin{tabular}{lll}
\hline Activity & Cost Slope $=\frac{\text { Crash Cost }- \text { Normal Cost }}{\text { Normal Time-Crash Time }}$ & Rank \\
\hline $1-2$ & $50(2)$ & IV \\
$1-3$ & $100(2)$ & VI \\
$2-4$ & $40(1)$ & III \\
$2-5$ & $60(5)$ & V \\
$3-4$ & $25(4)$ & II \\
$4-5$ & $10(2)$ & I \\
\hline
\end{tabular}

The other paths are $1 \rightarrow 2 \rightarrow 5 ; 1 \rightarrow 2 \rightarrow 4 \rightarrow 5 ; 1 \rightarrow 3 \rightarrow 4 \rightarrow 5$.

We rank the activities in ascending order of cost slope as given in the table then we crash activity $1-2$. It is the activity lying on the critical path with the minimum cost slope.

Step 5 .

\begin{tabular}{lllll}
\hline Normal duration & Crash activity & Crash cost & Indirect cost & Total cost \\
\hline 18 & - & - & 1260 & 1840 \\
17 & $1-2(1)$ & 50 & 1190 & 1820 \\
16 & $1-2(2)$ & 100 & 1120 & 1800 \\
15 & $2-5(1)$ & 160 & 1050 & 1790 \\
14 & $2-5(2)$ & 220 & 980 & 1780 \\
13 & $2-5(3)$ & 280 & 910 & 1770 \\
12 & $2-5(4)$ & 340 & 840 & 1760 \\
11 & $2-5(1-2-5) 4-5(1-3-4-5)$ & 410 & 770 & 1760 \\
\hline
\end{tabular}

Hence, the optimum project duration is 11 days and total cost Rs. 1760 . 


\section{Operation of trapezoidal NeUtrosophic FUZZY NUMBER IN SEQUENCING PROBLEM}

Sequencing problems are responsible for an appropriate selection of a sequence of jobs undertaken on a finite number of service facilities in some well defined technological order so as to optimize some efficiency measure such as total elapsed time or overall cost etc.

There steps the difficulty in solving a sequencing problem if there is an occurrence of uncertainty but the problem can be solved quiet easily using fewer efforts by applying the concept of impreciseness and its corresponding crispified value. In this field, we have taken a sequencing problem where there is an operation of three different machines and with the presence of a spectrum of different jobs to be satisfied \& undertaken. To turn the system into neutrosophic in nature, we consider the trapezoidal neutrosophic fuzzy number in the aspect of time in the database table.

\section{Problem definition}

We consider five different tasks each of which must go through the motor A, B, C respectively. Determine the sequence that will minimize the total elapsed time.

\begin{tabular}{llllll}
\hline Task No. & 1 & 2 & 3 & 4 & 5 \\
\hline \multirow{2}{*}{ Motor A } & $(2.5,4,5.5,7 ;$ & $(4.5,6,7.5,9 ;$ & $(3.5,5,6.5,8 ;$ & $(4,7,10,13 ;$ & $(4.4,5,5.5,6 ;$ \\
& $2.25,3.5,5,6.75 ;$ & $4.25,5.5,7,8.75 ;$ & $3.25,4.5,6,7.75 ;$ & $3.5,6,9,12.5 ;$ & $4.25,4.5,4.7,5.5 ;$ \\
& $3.5,5,6.5,8.5)$ & $5.5,7,8.5,10.5)$ & $4.5,6,7.5,9.5)$ & $6,9,12,16)$ & $4.45,5.05,5.20,5.45)$ \\
Motor B & $(0.75,1.5,2.25,3 ;$ & $(0.4,1,1.5,2 ;$ & $(1.5,3,4.5,6 ;$ & $(2.5,4.5,5,7 ;$ & $(1.75,2.5,3.25,4 ;$ \\
& $0.625,1.25,2,2.875 ;$ & $0.25,0.5,0.7,1.5 ;$ & $1.25,2.5,4,5.75 ;$ & $2.25,4,5,6.25 ;$ & $1.625,2.25,3,3.875 ;$ \\
& $1.25,2,2.75,3.75)$ & $0.45,1.05,1.20,1.445)$ & $2.5,4,5.5,7.5)$ & $3.5,5,6.5,8.5)$ & $2.25,3,3.375,4.75)$ \\
Motor C & $(1.75,2.5,3.25,4 ;$ & $(4.5,6,7.5,9 ;$ & $(4.4,5,5.5,6 ;$ & $(3.5,5,6.5,8 ;$ & $(4.5,6,7.5,9 ;$ \\
& $1.625,2.25,3,3.875 ; 4.25,5.5,7,8.75 ;$ & $4.25,4.5,4.7,5.5 ;$ & $3.25,4.5,6,7.75 ;$ & $4.25,5.5,7,8.75 ;$ \\
& $2.25,3,3.375,4.75)$ & $5.5,7,8.5,10.5)$ & $4.45,5.05,5.20,5.45)$ & $4.5,6,7.5,9.5)$ & $5.5,7,8.5,10.5)$ \\
\hline
\end{tabular}

\section{Step 1.}

In this sequencing problem we see that all the elements are Trapezoidal neutrosophic fuzzy member. First we convert this fuzzy number in to crisp number using our deneutrosophic value $R(\check{D}, 0)=$ $\frac{\left(a_{1}+b_{1}+c_{1}+d_{1}+e_{1}+f_{1}+g_{1}+h_{1}+i_{1}+j_{1}+k_{1}+l_{1}\right)}{12}$.

Thus the problem becomes.

\begin{tabular}{llllll}
\hline Task No. & 1 & 2 & 3 & 4 & 5 \\
\hline Motor A & 5 & 7 & 6 & 9 & 5 \\
Motor B & 2 & 1 & 4 & 5 & 3 \\
Motor C & 3 & 7 & 5 & 6 & 7 \\
\hline
\end{tabular}

\section{Step 2.}

The optimum sequence can be obtained by converting the problem into that of two-motors by the following steps $\operatorname{Max}\left(B_{i}\right)=5, \operatorname{Min}\left(A_{i}\right)=5$ Thus, $\operatorname{Min}\left(A_{i}\right) \geq \operatorname{Max}\left(B_{i}\right)$ is satisfied.

We define two motors $\mathrm{G}$ and $\mathrm{H}$ such that the processing time on $\mathrm{G}$ and $\mathrm{H}$ are given by,

$$
\mathrm{G}_{i}=\mathrm{A}_{i}+\mathrm{B}_{i} \text { and } \mathrm{H}_{i}=\mathrm{B}_{i}+\mathrm{C}_{i}, \quad i=1,2, \ldots, 5 .
$$

\begin{tabular}{llllll}
\hline Job & 1 & 2 & 3 & 4 & 5 \\
\hline Motor G & 7 & 8 & 10 & 14 & 8 \\
Motor H & 5 & 8 & 9 & 11 & 10 \\
\hline
\end{tabular}




\section{Step 3.}

We obtain the optimum sequence by using the steps of Johnson's Algorithm.

\begin{tabular}{lllll}
5 & 2 & 4 & 3 & 1 \\
\hline
\end{tabular}

\section{Step 4.}

\begin{tabular}{lllllllllll}
\hline \multirow{2}{*}{ Job } & \multicolumn{3}{c}{ Motor A } & \multicolumn{3}{c}{ Motor B } & \multicolumn{3}{c}{ Motor C } & \multicolumn{3}{c}{ Idle time } \\
\cline { 2 - 11 } & In & Out & In & Out & In & Out & A & B & C \\
\hline 5 & 0 & 5 & 5 & 8 & 8 & 15 & - & 5 & 8 \\
2 & 5 & 12 & 12 & 13 & 15 & 22 & - & 4 & - \\
4 & 12 & 21 & 21 & 26 & 26 & 32 & - & 8 & 4 \\
3 & 21 & 27 & 27 & 31 & 32 & 37 & - & 1 & - \\
1 & 27 & 32 & 32 & 34 & 37 & 40 & $40-32=8$ & 1 & - \\
& & & & & & & & $40-34=6$ & - \\
& & & & & & & 8 & 25 & 12 \\
\hline
\end{tabular}

Hence, Total Elapsed Time $=40 \mathrm{~h}$.

Idle time for motor $\mathrm{A}=8 \mathrm{~h}$; Idle time for motor $\mathrm{B}=25 \mathrm{~h}$ Idle time for motor $\mathrm{C}=12 \mathrm{~h}$.

Note. In case of trapezoidal neutrosophic fuzzy number Ye [41] consider score function as $S(\check{n})=$ $\frac{1}{3}\left(2+\frac{a+b+c+d}{4}-\frac{e+f+g+h}{4}-\frac{l+m+n+p}{4}\right)$ and accuracy function as $H(\check{n})=\frac{a+b+c+d}{4}-\frac{l+m+n+p}{4}$. If we consider these score function and accuracy function to convert a trapezoidal neutrosophic fuzzy number in to a crisp number for the above problem then we can find that all the processing time becomes negative in the table which is not possible as processing time in sequencing problem must be greater than zero. Thus, our de-neutrosophication method is a better approach.

\section{Conclusion}

There is a proliferating popularity for the conundrum concept of neutrosophic number presenting before the world a vibrant spice of logic and innovation to reach the zenith of excellence. The world is driven into a paradigm of brilliance as well as expertise with the formation and de-neutrosophication of the corresponding number which assists the researcher dealing with uncertainty and decision making problems. In this paper, we constructed the concept of neutrosophic number from different viewpoints and aspects for analyzing its true character. We have utilized the concept of linear and non linear form with the generalization of the pick value of truth, falsity, indeterminacy functions by considering trapezoidal neutrosophic numbers. We are furnished with the de-neutrosophication concept used in converting a neutrosophic number into a crisp number finding its significance for decision making problems. We solve a time cost optimization problem in trapezoidal neutrosophic fuzzy set environment which is done on sequencing problem giving a far ambidextrous result without any possible fiasco than the previous score function approach using the de neutrosophication method.

Consequently, we have inspected the following outcomes as:

- The generalized neutrosophic number and non linear neutrosophic number can be the better choice of decision makers rather than liner neutrosophic number.

- The concept of trapezoidal neutrosophic number is better than triangular neutrosophic number.

- The de-neutrosophication techniques namely (i) removal area method (ii) mean of interval method are is very useful to de-neutrsify the corresponding number.

- The numbers can be taken for different real life application model.

The work is done on trapezoidal nutrosophic number. But in future we can take the pentagonal, hexagonal and heptagonal neutrosophic number and de-neutrosophication can be made by different techniques. 
Thus, it can be concluded that the approach for taking the neutrosophic number is very helpful for the researchers who are involved in dealing the mathematical modeling with impreciseness in various fields of sciences and engineering. It reveals very realistic results in both mathematical points of view. There is still a massive amount of work in this field, hence many spectacular study can be explored with neutrosophic parameters.

\section{REFERENCES}

[1] M. Abdel-Basset, M. Mohamed, A.N. Hussien and A.K. Sangaiah, A novel group decision-making model based on triangular neutrosophicnumbers. Soft Comput. 15 (2017) 1-15.

[2] K. Atanassov, Intuitionistic fuzzy sets. Fuzzy Sets Syst. 20 (1986) 87-96.

[3] R. Bausys and B. Juodagalviene, Garage location selection for residential house by WASPAS-SVNS method. J. Civ. Eng. Manag. 23 (2017) 421-429.

[4] R. Bausys and E.K. Zavadskas, Multicriteria decision making approach by VIKOR under interval neutrosophic set environment. Econ. Comput. Econ. Cybern. Stud. Res. 49 (2015) 33-48.

[5] S. Broumi, A. Bakali, M. Talea, F. Smarandache and L. Vladareanu, Applying Dijkstra algorithm for solving neutrosophic shortest path problem. In: Proceedings on the International Conference on Advanced Mechatronic Systems. Melbourne, Australia (2016) 412-416.

[6] S. Broumi, M. Talea, F. Smarandache and A. Bakali, Single valued neutrosophic graphs: degree, order and size. In: Proceedings on IEEE International Conference on Fuzzy Systems (FUZZ). Vancouver, Canada (2016) 2444-2451.

[7] G. Büyüközkan and S. Güleryüz, Multi criteria group decision making approach for smart phone selection using intuitionistic Fuzzy TOPSIS. Int. J. Comput. Int. Syst. 9 (2016) 709-725.

[8] A. Chakraborty, S. Mondal, A. Ahmadian, N. Senu, D. De, S. Alam and S. Salahshour, Different forms of triangular neutrosophic numbers, de-neutrosophication techniques, and their applications. Symmetry 10 (2018) 327.

[9] A. Chakraborty, S. Mondal, A. Ahmadian, N. Senu, D. De, S. Alam and S. Salahshour, The pentagonal fuzzy number: its different representations, properties, ranking, defuzzification and application in game problem. Symmetry 11 (2018) 248.

[10] A. Chakraborty, S. Mondal, A. Ahmadian, N. Senu, D. De, S. Alam and S. Salahshour, Disjunctive representation of triangular bipolar neutrosophic numbers, de-bipolarization technique and application in multi-criteria decision-making problems. Symmetry 11 (2019) 932.

[11] K.P. Chaio, The multi-criteria group decision making methodology using type 2 fuzzy linguistic judgments. Appl. Soft Comput. 49 (2016) 189-211.

[12] S.J. Chuu, Selecting the advanced manufacturing technology using fuzzy multiple attributes group decision making with multiple fuzzy information. Comput. Ind. Eng. 57 (2009) 1033-1042.

[13] İ. Deli, A novel defuzzification method of SV-trapezoidal neutrosophic numbers and multi-attribute decision making: a comparative analysis. Soft Comput. 23 (2019) 12529.

[14] İ. Deli, Some operators with IVGSV TrN-numbers and their applications to multiple criteria group decision making. Neutrosophic Sets Syst. 25 (2019) 33-53.

[15] I. Deli and Y. Şubaş, A ranking method of single valued neutrosophic numbers and its applications to multi-attribute decision making problems. Int. J. Mach. Learn. Cybern. 8 (2016) 1309-1322.

[16] I. Deli and Y. Şubaş, Some weighted geometric operators with SVTrN-numbers and their application to multi-criteria decision making problems. J. Intell. Fuzzy Syst. 32 (2017) 291-301.

[17] I. Deli, M. Ali and F. Smarandache, Bipolar neutrosophic sets and their application based on multi-criteria decision making problems. In: 2015 International Conference on Advanced Mechatronic Systems (ICAMechS). IEEE, Beijing, China (2015).

[18] B. Efe, An integrated fuzzy multi criteria group decision making approach for ERP system selection. Appl. Soft Comput. 38 (2016) 106-117.

[19] M. Hanine, O. Boutkhoum, A. Tikniouine and T. Agouti, A new web-based framework development for fuzzy multi-criteria group decision-making. SpringerPlus 5 (2016) 601.

[20] P. Ji, H. Zhang and J. Wang, Selecting an outsourcing provider based on the combined MABAC-ELECTRE method using single-valued neutrosophic linguistic sets. Comput. Ind. Eng. 120 (2018) 429-441.

[21] S.S. Karaa and N. Cheikhrouhou, A multi criteria group decision making approach for collaborative software selection problem. J. Int. Fuzzy Syst. 26 (2014) 37-47.

[22] Y. Li, J. Wang and T. Wang, A linguistic neutrosophic multi-criteria group decision-making approach with EDAS method. Arab. J. Sci. Eng. 44 (2019) 2737-2749.

[23] R. Liang, J. Wang and H. Zhang, A multi-criteria decision-making method based on single-valued trapezoidal neutrosophic preference relations with complete weight information. Neural Comput. App. 30 (2018) 3383-3398.

[24] P. Liu and X. Liu, Multi attribute group decision making methods based on Linguistic intuitionistic Fuzzy power Bonferroni mean operators. Complex 2017 (2017) 1-15.

[25] F. Liu and X.H. Yuan, Fuzzy number intuitionistic fuzzy set. Fuzzy Syst. Math. 21 (2007) 88-91.

[26] S. Maity, A. Chakraborty, S. Dey, S. Mondal and S. Alam, A comprehensive study of a backlogging EOQ model with nonlinear heptagonal densefuzzyenvironment. RAIRO: OR 54 (2020) 267-286.

[27] X. Peng and C. Liu, Algorithms for neutrosophic soft decision making based on EDAS, new similarity measure and level soft set. J. Intell. Fuzzy Syst. 32 (2017) 955-968. 
[28] J.J. Peng, J.Q. Wang, X.H. Wu, H.Y. Zhang and X.H. Chen, The fuzzy cross-entropy for intuitionistic hesitant fuzzy sets and their application in multi-criteria decision-making. Int. J. Syst. Sci. 46 (2014) 2335-2350.

[29] J.J. Peng, J.Q. Wang, X.H. Wu, J. Wang and X.H. Chen, Multi-valued neutrosophic sets and power aggregation operators with their applications in multi-criteria group decision-making problems. Int. J. Comput. Intell. Syst. 8 (2015) $345-363$.

[30] J.J. Peng, J.Q. Wang, J. Wang, H.Y. Zhang and X.H. Chen, Simplified neutrosophic sets and their applications in multi-criteria group decision making problems. Int. J. Syst. Sci. 47 (2016) 2342-2358.

[31] J. Peng, J.-Q. Wang, J.-H. Hu and C. Tian, Multi-criteria decision-making approach based on single-valued neutrosophic hesitant fuzzy geometric weighted choquet integral heronian mean operator. J. Intell. Fuzzy Syst. 35 (2018) 3661-3674.

[32] H. Pouresmaeil, E. Shivanian, E. Khorram and H.S. Fathabadi, An extended method using TOPSIS and VIKOR for multiple attribute decision making with multiple decision makers and single valued neutrosophic numbers. Adv. Appl. Stat. 50 (2017) 261-292.

[33] F. Shen, J. Xu and Z. Xu, An outranking sorting method for multi-criteria group decision making using intuitionistic fuzzy sets. Inf. Sci. 334 (2016) 338-353.

[34] F. Smarandache, A Unifying Field in Logics Neutrosophy: Neutrosophic Probability, Set and Logic. American Research Press, Rehoboth (1998).

[35] J.Q. Wang and X.E. Li, The TODIM method with multi-valued neutrosophic sets. Control Decis. 30 (2015) 1139-1142.

[36] H.B. Wang, F. Smarandache, Y.Q. Zhang, R. Sunderraman, Single-valued neutrosophic sets. Multispace Multistructure 4 (2010) 410-413.

[37] J.Q. Wang, J.J. Peng, H.Y. Zhang, T. Liu and X.H. Chen, An uncertain linguistic multi-criteria group decision-making method based on a cloud model. Group Decis. Negotiation 24 (2015) 171-192.

[38] X. Wu, J. Wang, J. Peng and J. Qian, A novel group decision-making method with probability hesitant interval neutrosphic set and its application in middle level manager's selection. Int. J. Uncertainty Quantificat. 8 (2018) 291-319.

[39] J. Ye, Prioritized aggregation operators of trapezoidal intuitionistic fuzzy sets and their application to multicriteria decision making. Neural Comput. App. 25 (2014) 1447-1454.

[40] J. Ye, A multicriteria decision-making method using aggregation operators for simplified neutrosophic sets. J. Intell. Fuzzy Syst. 26 (2014) 2459-2466.

[41] J. Ye, Trapezoidal neutrosophic set and its application to multiple attribute decision-making. Neural Comput. App. 26 (2015) $1157-1166$.

[42] J. Ye, Exponential operations and aggregation operators of interval neutrosophic sets and their decision making methods. SpringerPlus 5 (2016) 1488.

[43] X. You, T. Chen and Q. Yang, Approach to multi-criteria group decision-making problems based on the best-worst-method and ELECTRE method. Symmetry 8 (2016) 95.

[44] L.A. Zadeh, Fuzzy sets. Inf. Control 8 (1965) 338-353.

[45] E.K. Zavadskas, R. Bausys, A. Kaklauskas, I. Ubarte, A. Kuzminske and N. Gudiene, Sustainable market valuation of buildings by the single-valued neutrosophic MAMVA method. Appl. Soft Comput. 57 (2017) 74-87. 